\title{
Cholecystocutaneous Fistula Secondary to Chronic Calculous Cholecystitis
}

\section{S. ljaz S. Lidder W. Mohamid H.H. Thompson}

Department of General and Colorectal Surgery, Lister Hospital, Stevenage, UK

\section{Key Words}

Cholecystocutaneous fistula $\cdot$ Chronic cholecystitis

\begin{abstract}
Spontaneous cholecystocutaneous fistula is an exceptionally unusual complication of chronic calculous cholecystitis now. The remarkable drop in incidence is probably associated with the introduction of antimicrobial therapy and early surgical management of biliary tract disease. We report a case of spontaneous cholecystocutaneous fistula in a patient who presented with an abscess in the right upper quadrant.
\end{abstract}

\section{Case Report}

A 74-year-old woman presented as an emergency with a several day history of fevers, malaise and a right subcostal mass. She had no significant past medical history; however, she had a negative laparotomy following a road traffic accident many years previously.

On examination, the patient was apyrexial with a $7 \times 3 \mathrm{~cm}$ tender, erythematous mass palpable over the right upper quadrant. The white cell count on admission was $10.0 \times 10^{9} / 1$, C-reactive protein was 68 $\mathrm{mg} / \mathrm{l}$ and liver function tests were normal apart from a marginally deranged alkaline phosphatase of 121 $\mathrm{IU} / \mathrm{l}$ and a gamma glutamyl transferase of $50 \mathrm{IU} / \mathrm{l}$.

A subsequent CT scan of the abdomen confirmed the clinical suspicion of a superficial abscess, which extended superiorly to the costal margin and inferiorly to the right iliac crest. No other abnormality was demonstrated apart from the incidental finding of a simple cyst on her right kidney.

The abscess discharged spontaneously, the pus culture from it revealed coliforms but tissue from a needle core biopsy demonstrated inflammatory changes only. The patient was discharged only to return 2 months later with a recurrent abscess that was then formally incised and drained. Again, biopsies revealed chronic inflammatory changes only and there was no evidence of tuberculosis either on biopsies or tissue culture. As the wound was slow to heal, a further CT scan was performed which suggested a communication between the gallbladder and the abscess cavity (fig. 1 ).

At subsequent laparotomy, a very fibrotic and inflamed gallbladder, full of large cholesterol stones, was found to be adherent to the anterior abdominal wall via a fistulous track. This track was divided, thereby freeing the gallbladder from the abdominal wall and then a retrograde cholecystectomy was carried out. The fistulous track was enlarged, curetted out and irrigated thoroughly with a betadine 


\begin{tabular}{c|l|l|l}
$\begin{array}{c}\text { Case Reports in } \\
\text { Gastroenterology }\end{array}$ & $\begin{array}{l}\text { Case Rep Gastroenterol 2008;2:71-75 } \\
\text { D0I: } 10.1159 / 000118831\end{array}$ & Published online: March 11, 2008 & $\begin{array}{l}@ 2008 \text { S. Karger AG, Basel } \\
\text { ISSN 1662-0631 } \\
\text { www.karger.com/crg }\end{array}$ \\
\hline
\end{tabular}

saline solution. Then the cavity was loosely packed with an alginate dressing and left to heal by secondary intention. The patient recovered without incident and was discharged home. Histological examination of the gallbladder confirmed chronic cholecystitis (fig. 2 , fig. 3 ).

\section{Discussion}

The incidence of complications like cholecystocutaneous fistulae has fallen dramatically since the advent of intravenous antibiotics and the emergence of elective and emergency cholecystectomies for gallbladder disease, such that now it is seen as a vanishingly rare phenomenon. There have been fewer than 25 reports in the medical literature over the past half-century.

Spontaneous perforation of calculous cholecystitis to abdominal viscera is not uncommon, particularly to the duodenum and colon, and it has also been seen in the bronchial tree, stomach and urinary tract [1].

Cholecystocutaneous fistulae typically present in the elderly female, as a painless draining sinus tract in the right upper quadrant of the abdomen, but this tract may alternatively drain to the right iliac fossa, right groin, right gluteal region, umbilicus or left upper quadrant. There is usually a history of chronic biliary tract disease, however patients generally do not report a previous distinct episode of acute cholecystitis [2].

Increased intraluminal pressure in the gallbladder secondary to calculous obstruction is thought to impair blood flow and lymphatic drainage, thereby leading to mural necrosis and perforation, which can either be an acute or indolent process. Acutely, gallbladders may perforate, leading to peritonitis or an abscess around the gallbladder. A chronic perforation can lead to an internal or external biliary fistula and these arise most commonly from the fundus of the gallbladder.

CT abdominal scans and/or fistulograms are usually necessary to make the diagnosis. A combination of microbiology, CT of the abdomen and laparoscopy were sufficient in this case.

Following control of the acute inflammatory process in the abdominal wall, an elective cholecystectomy is advisable in these patients. An open approach is usually favoured, although a laparoscopic technique has been described by Kumar [3].

In conclusion, this case underlines the fact that all patients presenting with right-sided abdominal wall abscesses need to be investigated thoroughly for underlying pathology, particularly on a background of calculous biliary tract disease. 
Fig. 1. Abdominal computed tomography scan. Arrow points to the likely cholecystocutaneous fistulous track.

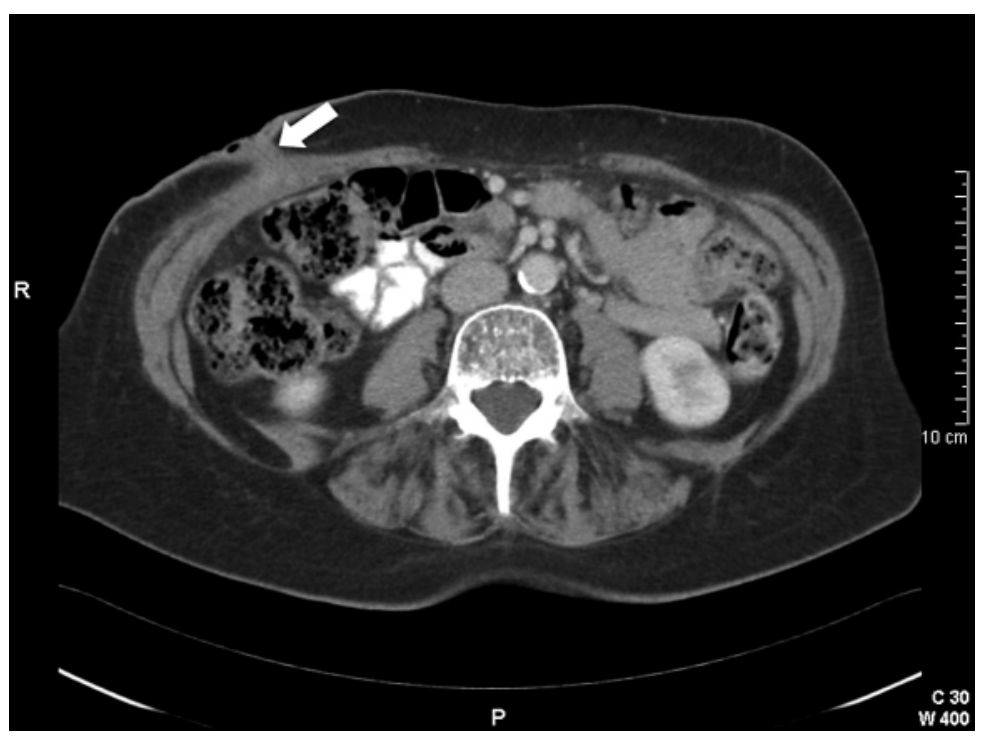

Fig. 2. Gallbladder mucosa exhibiting features of chronic cholecystitis. Haematoxylin and eosin stain.

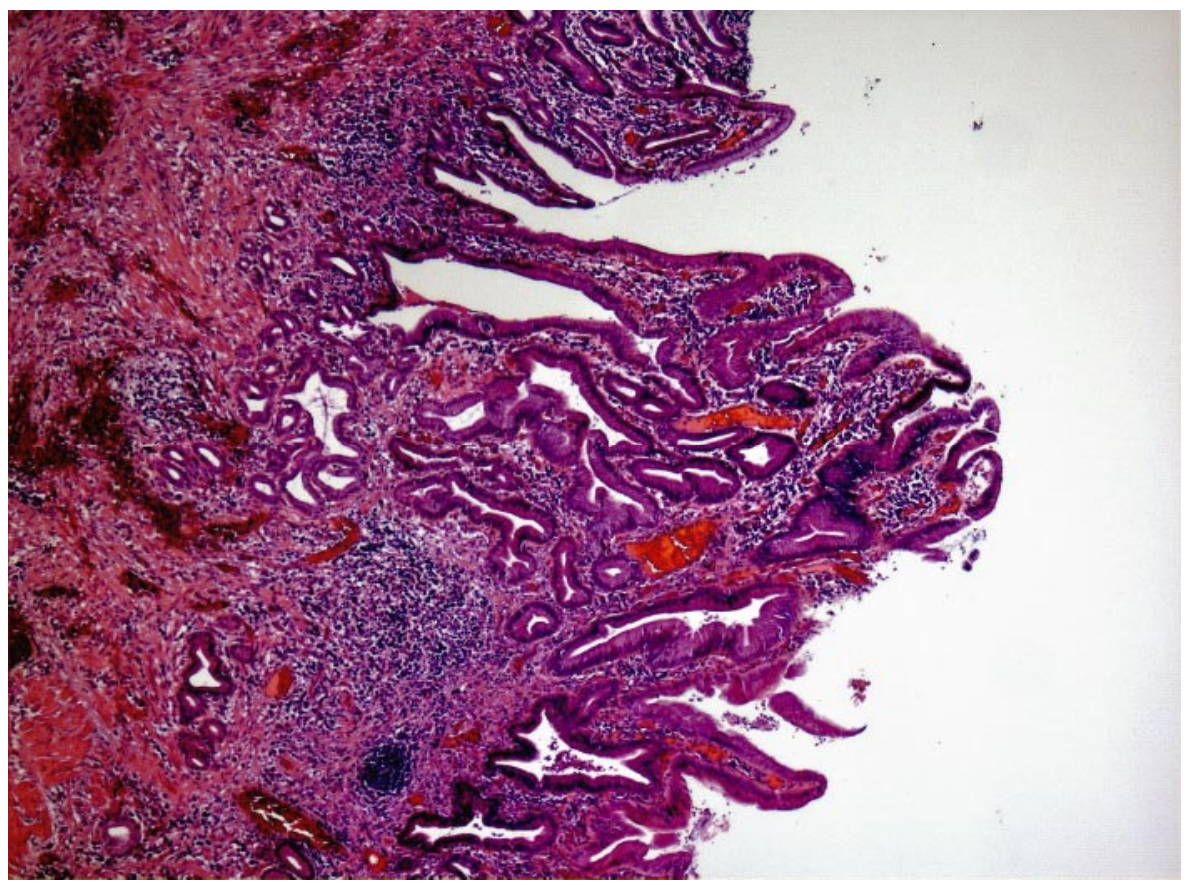


Fig. 3. Opening of the gallbladder fistula. Haematoxylin and eosin stain.

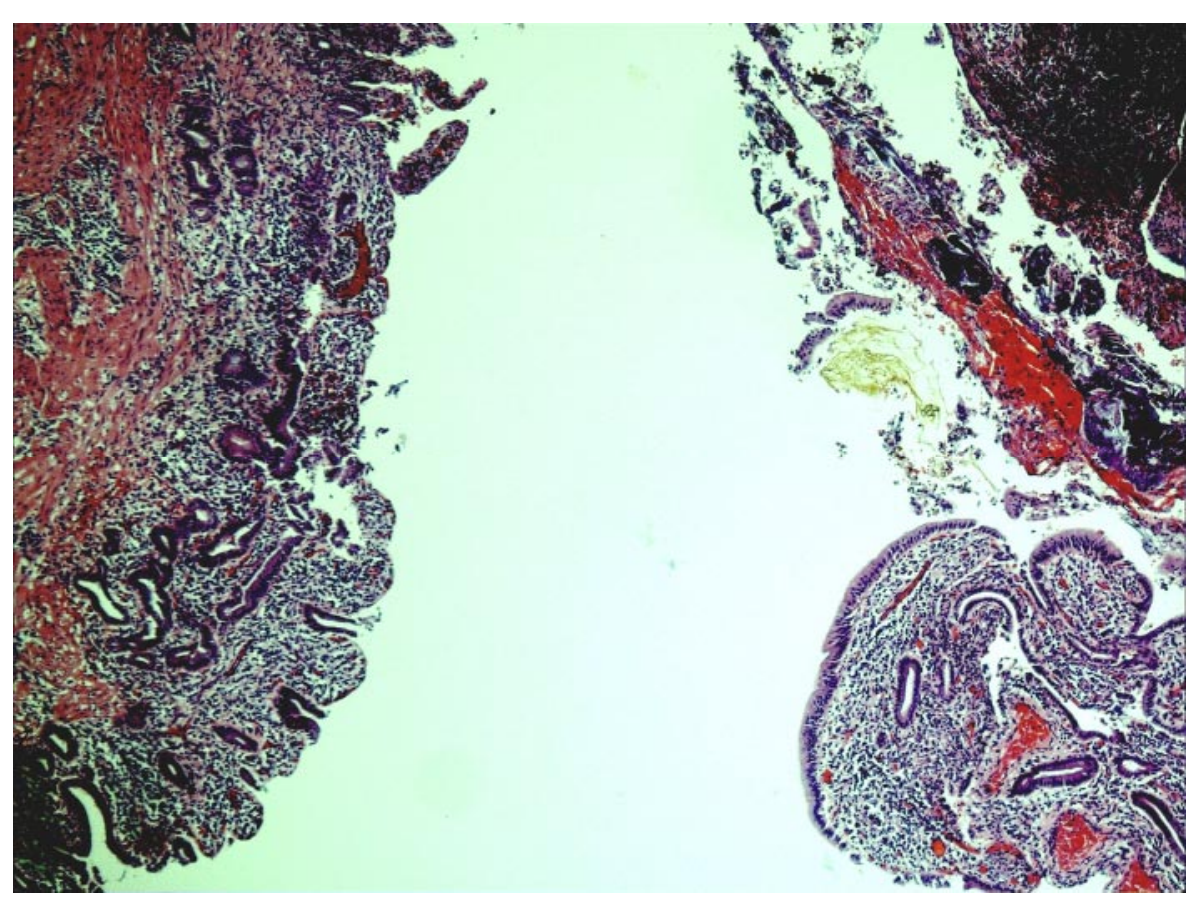




\section{References}

1 Urban CA, Lima RS, Blegii-Torres LF: Spontaneous combined internal and external biliary fistulae in association with gallstones and gliomatosis of the gallbladder. Rev Bras Cancerol 2000;47:273-276.

-2 Nicholson T, Born MW, Garber E: Spontaneous cholecystocutaneous fistula presenting in the gluteal region. J Clin Gastroenterol 1999;28:276-277.

-3 Kumar SS: Laparoscopic management of cholecystocutaneous abscess. Am Surg 1999;64:1192-1194. 